\title{
Selection of Nonstationary Dynamic Features for Obstructive Sleep Apnoea Detection in Children
}

\author{
L. M. Sepulveda-Cano, ${ }^{1}$ E. Gil, ${ }^{2}$ P. Laguna, ${ }^{2}$ and G. Castellanos-Dominguez ${ }^{1}$ \\ ${ }^{1}$ Grupo de Procesamiento y Reconocimiento de Señaales, Universidad Nacional de Colombia, Km. 9, Vía al Aeropuerto, \\ Campus La Nubia, 17001000 Manizales, Colombia \\ ${ }^{2}$ Communications Technology Group (GTC), Aragón Institute of Engineering Research (I3A), ISS, University of Zaragoza, CIBER-BBN, \\ María de Luna 1, 50018 Zaragoza, Spain \\ Correspondence should be addressed to L. M. Sepulveda-Cano, lmsepulvedac@bt.unal.edu.co
}

Received 1 July 2010; Revised 6 December 2010; Accepted 26 January 2011

Academic Editor: Antonio Napolitano

Copyright (๑) 2011 L. M. Sepulveda-Cano et al. This is an open access article distributed under the Creative Commons Attribution License, which permits unrestricted use, distribution, and reproduction in any medium, provided the original work is properly cited.

This paper discusses the methodology for selecting a set of relevant nonstationary features to increase the specificity of the obstructive sleep apnea detector. Dynamic features are extracted from time-evolving spectral representation of photoplethysmography envelope recordings. In this regard, a time-evolving version of the standard linear multivariate decomposition is discussed to perform stochastic dimensionality reduction. For training aim, this work analyzes the concrete set comprising filter banked dynamic features that include spectral centroids, the cepstral coefficients as well as their timevariant energies. Performance of classifier accuracy is provided for the collected polysomnography recordings of 21 children. Moreover, since the apnea diagnosing is based on analysis of set of fragments partitioned from the photoplethysmography envelope recordings, a new approach for their indirect labeling is described. As a result, performed outcomes of accuracy bring enough evidence that if using a subset of cepstral-based dynamic features, then patient classification accuracy can reach as much as $83.3 \%$ value, when using a $k$-nn classifier, as well. Therefore, photoplethysmography-based detection provides an adequate scheme for obstructive sleep apnea diagnosis.

\section{Introduction}

Regarding the diagnosis of obstructive sleep apnea (OSA) syndrome, which is characterized by recurrent airflow obstruction caused by total or partial collapse of the upper airway, several strategies have been developed to decrease the number of the sleep recordings needed for usually performed polysomnography [1] that is related as an expensive and time-consuming procedure. One promising alternative is the pulse photoplethysmography signal (PPG) that is a simple, but useful, method for measuring the pulsatile component of the heartbeat. PPG measurement evaluates peripheral circulation, and is tie related either to arterial vasoconstriction or vasodilatation generated by the autonomic nervous system, being modulated by the heart cycle. Furthermore, automatic detection of time-variant decreases in the amplitude fluctuations of PPG have shown their utility for OSA diagnosis [2-4].

Nonetheless, since there is a large number of situation when PPG enveloped is affected independently of the apnoea status, then, a low ratio sensitivity/specificity is accomplished. Therefore, to better discriminate between apnoea from other PPG envelop alterations an improved set of representing features should be taken into account, particularly, stochastic modeling of dynamic features for OSA detection is to be further considered in this work.

The use of stochastic modeling, when taking into account evolution of random biological variables along time (herein referred as dynamic features) precedes the necessity of building a proper methodology of their processing. Furthermore, it is well known that the complexity of stochastic modeling increases because of need to carry out 
the adequate nonstationary estimation of parameters derived from biosignal recordings. One can refer to that issue as the most important difference between static and dynamic statistical processing.

As a rule, methodology for analysis of time series is based on the assumption that there is always a processing time window of such a length that the piecewise stationarybased approach of analysis holds. Although determination of proper stationary data length remains as an open issue. With this in mind, the time-frequency representation (TFR) has been proposed before for the analysis of nonstationary biomedical data. Among the most popular TFR used to investigate the dynamic properties of the time-evolving spectral parameters, during either transient physiological or pathological episodes, are those computed directly from the raw data after preprocessing, termed nonparametric approaches. Specifically, the Wavelet Transform (WT) and he Short Time Fourier Transform (STFT) are commonly used. Though the former TFR is likely to avoid the $t$ - $f$ resolution compromise, the latter nonparametric approach is desirable for biosignals with a slow time varying spectrum [5], as it is the case for PPG recordings. However, the application of TFR to the analysis of short transient signals (like in case of PPG envelope) is a complex, and difficult task due to the inherent limitations of the TFR techniques for extracting the relevant, but not redundant characteristics. In other words, without accurate models to describe properly the dynamic behavior of PPG envelope biosignals, the use of $t$ - $f$ processing methods, based on stochastic assumptions, may fail to provide satisfactory results. In this sense, it has been established the discriminating capability of frequency bands of biological activity between normal and pathological patterns, and for that reason, the set of TFR-based stochastic features to be considered should be suitable estimated by time-evolving spectral subband methods.

Nonetheless, the amount of measured time-variant features can be large, no mentioning that the sampling rate used for these measurements may be also high. Assuming that dynamic variables are low-pass processes, then the enclosed information within the stochastic data becomes highly correlated. This fact provides large data-sets holding big amount of redundancy, which in turn leads to either overtraining data or significant increasing of computational overhead. In such a situation, dimension reduction that should be strongly considered might determine the adequate number of relevant features to select either by encoding or removing both redundant and irrelevant information. Furthermore, the concept of biosignal interpretation becomes critical, whose ultimate goal is the proper classification of the features, but also to depict them in order to maximize correct interpretation and physiological or clinical meaning [6].

Extraction of relevant stochastic information from dynamic feature sets has been discussed in the past, as a means to improve performance during and after training in learning processes. Thus, to get an effective feature selection algorithm, in the context of an inference, two main issues are to be overcame [7]: the same measure associated to a given relevance function (i.e., a proper measure of distance for time series), and the multivariate transformation through the time axis, which is assumed to maximize the measure of relevance present in the nonstationary features by their projection onto a new space. For a dimension reduction, statistical latent variable techniques can be applied, for example, by using Principal Component Analysis (PCA) that maximizes the variability on the input data set. This specific and unique property of PCA makes the stationary signals easy to interpret. But standard latent variable techniques clearly do not take into consideration the timeevolving nature of random biological variables, since they are grounded on a common representation that minimizes the global reconstruction error.

The aim of this study is to select a set of relevant nonstationary features, extracted from $t-f$ representation of time-dependant PPG envelope signals, to increase the specificity in the apnoea detector. This work analyzes the set comprising filter banked dynamic features that includes spectral centroids as well as the cepstral coefficients. Specifically, a time-evolving version of the standard linear multivariate decomposition is discussed throughout this paper to perform stochastic dimensionality reduction of the dynamic features in hand. The rest of the paper is organized as follows: Section 2 introduces materials and methods focused on generation of nonstationary features, extracted from $t$ - $f$ representation of time-dependant PPG envelope signals. Also, the proposed methodology of stochastic training is evaluated using real PPG recordings. The attained results are discussed in Section 5. Finally, Section 6 presents the conclusions and discusses some possibilities for future work.

\section{Materials and Methods}

2.1. Generation of Enhanced Dynamic Features. The PPG envelope, $y(t)$, is estimated based on the root mean square series of input PPG signal, $y_{\mathrm{PPG}}(t)$. So, the discrete version of PPG envelope, after mean removal by a moving average filter, can be written as follows [2]:

$$
y(n)=\sqrt{\frac{1}{N} \sum_{k=n-(N-1)}^{n}\left(y_{\mathrm{PPG}}(k)-\frac{1}{M_{l=k-(M-1)}} \sum_{\mathrm{PPG}}^{k}(l)\right)^{2}}
$$

where the values for the window length of the filtering, $M$, and the root mean square series, $N$, are fixed to be 25 and twice the mean cardiac cycle, respectively.

Generally, a direct way of describing the PPG envelope, $y(t)$, in both time and frequency $(t-f)$ domains becomes its time-evolving spectral representation. Thus, for estimating TFR of random signals, power spectral density is commonly used, which for a given biosignal, $y(t)$, is directly represented by the spectrogram:

$$
\begin{array}{r}
\mathbf{S}_{y}(t, f)=\left|\int_{T} y(\tau) \phi(\tau-t) e^{-j 2 \pi f \tau} d \tau\right|^{2} \\
t, \tau \in T, \quad \mathbf{S}_{y}(t, f) \in \mathbb{R}^{+} .
\end{array}
$$


Supported on classical Fourier Transform, the Short Time version (termed STFT) introduces a time localization concept by using a tapering window function of short duration, $\phi$, that is, going along the studied biosignal, $y(t)$.

Extracted from the spectrogram-based TFR, any stochastic feature $x(t)$ refers to random numeric values comprising measures evolving over time, that is, there is a certain set of parameters, $\Xi=\left\{\mathbf{x}_{i}=x_{i}(t): i=1, \ldots, p\right\}$, that are changing along the time axis, $t \in T$, is supposed to carry temporal information of the nonstationary biosignals. In this regard, some nonparametric TFR-based dynamic measures have been widely accepted, mainly, those estimated by spectral subband methods, when efficiently combining frequency and magnitude information from the short-term power spectrum of the input biosignals. For instance, given a discrete time series, $y(n)$, being the sampled version of a continuous biosignal recording $y(t)$, the set of Linear Frequency Cepstral Coefficients (LFCC) is proposed to be employed, which is extracted by Discrete Cosine Transform of triangular logfilter banks, $\left\{F_{m}(k): m=1, \ldots, n_{M}\right\}$, linearly spaced in the frequency domain:

$$
x_{n}(l)=\sum_{m=1}^{n_{M}} \log \left(s_{m}(l)\right) \cos \left(n\left(m-\frac{1}{2} \frac{\pi}{p}\right)\right),
$$

where $p$ is the number of desired LFCC features to be considered, and $s_{m}(l)$ is the weighted sum of each frequency filter response set, $s_{m}(l)=\sum_{k=1}^{n_{K}} S_{y}(l, k) F_{m}(k)$, with $m, l$, and $k$ being the indexes for filter ordinal, time, and frequency axes, respectively; $n_{K}$ stands for the number of samples in the frequency domain. Other effective way of generating $t$ - $f$ based time-variant features is achieved through computation of the histograms of the subband spectral centroids that are estimated for each filter in the frequency domain, $F_{m}^{\prime}(k)$, by

$$
x_{n}(l)=\frac{\sum_{k=1}^{n_{K}} k F_{n}^{\prime}(k) S_{y}^{\gamma}(l, k)}{\sum_{k=1}^{n_{K}} F_{n}^{\prime}(k) S_{y}^{\gamma}(l, k)},
$$

where $\gamma$ is a parameter representing the dynamic range of the spectrum that is used for computation of the centroid. The filters $F_{n}^{\prime}(k)$ are linearly distributed along the spectrum. In addition, the energy around each centroid can be also considered as time-variant feature that for a fixed bandwidth $\Delta k$ is computed by means of

$$
x_{n}(l)=\sum_{k=\hat{x}_{n}(l)-\Delta k}^{\hat{x}_{n}(l)+\Delta k} S_{y}(l, k)
$$

where $\hat{x}_{n}(l)$ is the actual value of the time-variant centroid that is estimated by (4).

2.2. Relevance Analysis of Stochastic Features. Because of high computational cost of stochastic feature-based training, dimension reduction of input spaces is to be carried out, being latent variable techniques widely used for this aim that finds a transformation reducing $p$-dimensional stochastic feature arrangement, $\boldsymbol{\Xi} \in \mathbb{R}^{p \times T}$, into $q$-dimensional stochastic set, $\mathbf{Z} \in \mathbb{R}^{q \times T}, q \leq p$, in such a way that the data information is maximally preserved. Besides, as the relevance function, $g \in \mathbb{R}$, the evaluation measure of transformation is given that distinguishes variables effectively representing the subjacent physiological phenomena, termed relevant stochastic features.

The set of stochastic features, $\left\{\mathbf{x}_{i}\right\}$, is represented by the observation assemble comprising $N$ objects that are disposed in the input observation matrix $\mathbf{X}_{\Xi}=\left[|| X_{1}|\cdots| X_{i}|\cdots| X_{N} \mid\right]$. In turn, every object, denoted as $X_{i}, i=1, \ldots, N$, is described by the respective observation set of timevariant arrangements, $\left\{\mathbf{x}_{j i} \subset \boldsymbol{\Xi}, j=1, \ldots, p\right\}$, such that, $X_{i}=\left[\left|\mathbf{x}_{1 i}\right| \cdots\left|\mathbf{x}_{j i}\right| \cdots\left|\mathbf{x}_{p i}\right|\right]^{\top}, X_{i} \in \mathbb{R}^{p \times T}$, where $\mathbf{x}_{j i}=$ $\left[x_{j i}(1) \cdots x_{j i}(t) \cdots x_{j i}(T)\right]$ is each one of the measured or estimated short-term features from biosignal recordings, equally sampled evolving through the time, and being $x_{i j}(t)$, the $j$ th stochastic feature for the $i$ th object upon a concrete $t$ instant of time.

For the sake of simplicity, the reduction dimension is developed when projecting by the simplest time-evolving latent variable approach, that is, time-adapted PCA. So, given the observation matrix, $\mathbf{X}_{\boldsymbol{\Xi}}$, there will be a couple of orthonormal matrixes, $\mathbf{U} \in \mathbb{R}^{N \times N}, \mathbf{V} \in \mathbb{R}^{p T \times p^{T}}$, plus diagonal matrix $\Sigma_{\mathbf{X}}$, as well, so that a simple linear decomposition takes place, that is, $\mathbf{X}_{\boldsymbol{\Xi}}=\mathbf{U} \boldsymbol{\Sigma}_{\mathbf{X}} \mathbf{V}^{\top}$, where $\boldsymbol{\Sigma}_{\mathbf{X}} \in \mathbb{R}^{p T \times p^{T}}$ holds first ordered $q$ as most relevant eigenvalues of matrix $\mathbf{X}_{\Xi}$, $v_{1} \geqslant v_{2}, \ldots, \geqslant v_{q} \geqslant v_{q+1}, \ldots, \geqslant v_{p T} \geqslant 0$, that implies the relevance measure to be considered. The minimum mean squared-based error is assumed as the evaluation measure of transformation, $g\left(\mathbf{X}_{\boldsymbol{\Xi}}, \mathbf{Z}\right) \sim \min \mathcal{E}\left\{\|\boldsymbol{\Xi}-\mathbf{Z}\|_{2}\right\}$, (where $\|\cdot\|_{2}$ is the norm squared value, and $\mathscr{E}\{\cdot\}$ is the is the expectance operator), that is, maximum variance is preferred as relevance measure, when the following estimation of covariance matrix is carried out:

$$
\operatorname{cov}\left\{\mathbf{X}_{\Xi}\right\}=\mathbf{X}_{\Xi}^{\top} \mathbf{X}_{\Xi}=\mathbf{V} \Sigma_{\mathbf{X}}^{2} \mathbf{V}^{\top}
$$

To make clear the contribution of each time-variant value $x_{i j}(t)$, expression (6) can be further extended in the form:

$$
\mathbf{X}_{\Xi}^{\top} \mathbf{X}_{\Xi}=\sum_{j=1}^{p} v_{j}^{2} V_{j} V_{j}^{\top},
$$

where $V_{j}$ is the $j$ th column of matrix $\mathbf{V}$.

Consequently, the amount of relevance captured at every moment $t$ by the singular value decomposition, that is associated to the whole set of features is assessed as the following time-variant relevance measure:

$$
g\left(\mathbf{X}_{\Xi}, \mathbf{Z} ; t\right)=\sum_{j=1}^{q}\left|v_{j}^{2} V_{j}\right|
$$

Therefore, the proper selection of the most relevant stochastic features containing essential information can be achieved if choosing the truncated set of extracted from TFR parameters that exhibit the higher time-variant values of variance-based relevance measure. In other words, dimension reduction is carried out by adapting in time commonly used latent variable techniques (by example, 


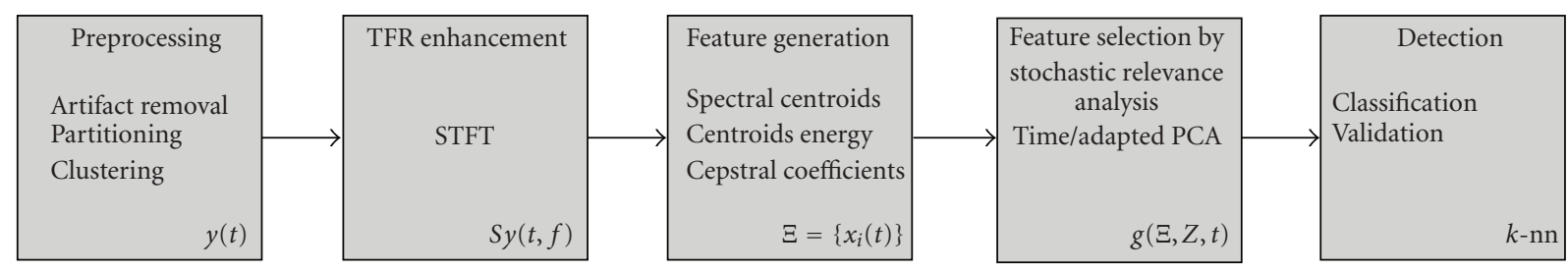

FIGURE 1: Schematic representation of an automated system for OSA diagnosing from $t$ - $f$ representation of PPG envelope.

the one expressed by (6)), in such a way, that the data information is maximally preserved, given a relevance function as evaluation measure of time-variant transformation, and therefore, distinguishing relevant stochastic features.

\section{Experimental Setup}

Based on relevance analysis of dynamic features that are extracted from $t$ - $f$ representation of PPG envelope, the proposed methodology for diagnosing obstructive sleep apnoea appraises next stages (see schematic representation of Figure 1): (a) preprocessing, (b) enhancement of TFR, (c) dynamic feature extraction embracing dimension reduction of TFR-derived time series, and (d) OSA detection.

3.1. Clinic Photoplethysmography Database. This study uses the collection of polysomnography recordings of 21 children that were acquired over all-night-long sessions, as detailedly described in [3]. The children aging within $4.5 \pm 2$ years were referred to the Miguel Servet Children's Hospital in Zaragoza for suspected sleep-disordered breathing. Electroencephalographic electrode positions C3, C4, O1, and $\mathrm{O} 2$, chin electromyogram, electrocardiographic leads I and II, eye movements, airflow as well as chest and abdominal respiratory efforts were recorded by a digital polygraph (BITMED EGP800), according to the standard procedure of the American Thoracic Society [8]. PPG and arterial oxygen saturation $\left(\mathrm{SaO}_{2}\right)$ were measured continuously using a pulse oximeter (COSMO ETCO2/SpO2 Monitor Novametrix, Medical Systems). Recordings were stored with a sample rate of $100 \mathrm{~Hz}$, except electrocardiographic biosignals that were sampled at $500 \mathrm{~Hz}$. OSA evaluation from PSG data were scored by clinical experts using the standard procedures and criteria given in [9]. Children often desaturate with short apneas, as they have a lower functional residual capacity and a faster respiratory rate than adults. Therefore, obstructive apneas of any length are scored when interpreting pediatric sleep studies, as compared with the 10-second duration in adults. Children may develop clinical sequelae with what appears to be relatively mild OSA. Thus, an apnea index of 10 is considered to be severe by most pediatric pulmonologists, whereas it is considered only mildly abnormal in adults. One reason why a low apnea index can be associated with severe clinical disease is that the apnea index, the parameter used most often to characterize disordered breathing in adults, does not give an accurate picture of the nature of the breathing disturbance in children
[10]. Thus, ten children were diagnosed with OSA, whereas the remaining eleven were diagnosed as normal.

3.2. Artifact Removal. It has been established that PPG measurements are quite sensitive to patient and/or probetissue movement artifact. Removal of such motion artifact as well as its separation from proper quality, although highly variable, pulse recordings is a nontrivial signal processing exercise [11]. To cope with this drawback, the artifact Hjorth detector is used. The principle behind the detector is that when the PPG signal differs largely from an oscillatory signal, it is very likely an artifact. Hjorth parameter has been proposed as an estimation of the central frequency of a signal and as half of the bandwidth. Further details of used artifact removal procedure are explained in [2].

3.3. Labeling of PPG Envelope Recordings. It is worth noting that the discussed automated system for OSA diagnosing is based on analysis of set of fragments that are partitioned from the PPG envelope recordings. In particular, once the OSA diagnostic labeling of PSG recording database had been made by experts after clinical analysis of the considered children patient group, then, all recordings that in average can last as much as 8 hours are firstly partitioned into fragments of two different considered lengths: 15 or 60 minutes. Each fragment of either length is labeled using a decision rule based on $\mathrm{SaO}_{2}$ signal which had been simultaneously measured in time. Moreover, because of computational load the fragments are partitioned again into segments lasting 90 seconds. Each 90-second frame is given the same label of the respective PPG fragment from where the segment has been extracted. So, labeling of partitioned PPG envelope recordings is provided according to the following procedures.

(1) Fragment Labeling. In general, pathologic patients can have some time periods related to both apneas and oxygen desaturation, but, they can also exhibit some normal periods without any respiratory problems. So, regarding subject diagnosis, it is useful to consider PSG fragments as a whole entity, then, a subject classification is carried out based on the number of PSG fragments that are related to apneic periods. The length of considered fragments is a tradeoff between fragments and subject classification. In this study, both 15-minute and 1-hour PSG fragments are considered, as recommended in [3]. This assessed set of PSG fragments is labeled as follows. 


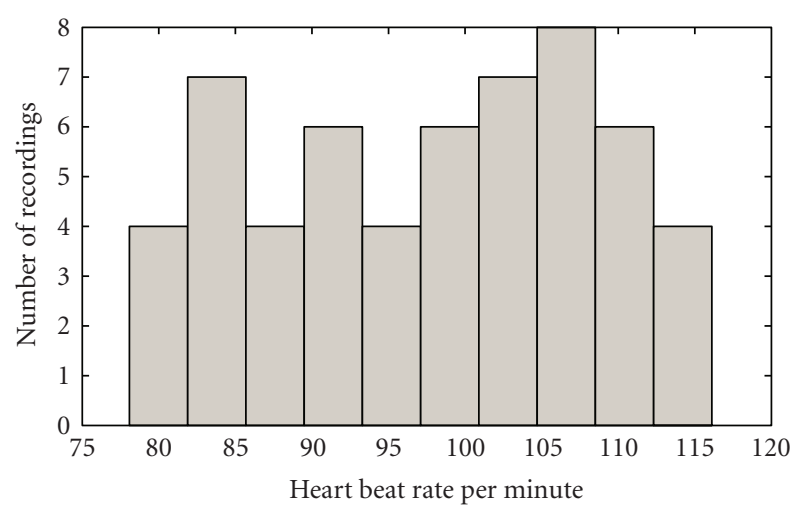

FIGURE 2: Histogram of heart beat rate per minute for a given set of labeled PPG fragments.

At the beginning, a baseline level $\beta$, is fixed for each patient that is related to the oxygen saturation, which corresponds to the $\mathrm{SaO}_{2}$ signal mode of the entire night recording. Then, the total time intervals with $\mathrm{SaO}_{2}$ signal below $\beta-$ $3 \%, t_{\beta-3}$ are calculated for each PSG fragment. Polysomnographic fragments of either length, 15-minute or 1-hour, are labeled according to the following criteria:

$$
\begin{array}{ll}
t_{\beta-3}<0.9 \text { minutes, } & \text { control, } \\
0.9 \text { minutes }<t_{\beta-3}<3 \text { minutes, } & \text { doubt, } \\
t_{\beta-3}>3 \text { minutes, } & \text { pathologic. }
\end{array}
$$

The above imposed criteria imply a minimum of $5 \%$ of the time with evident oxygen desaturation to be considered as pathologic. The assumed threshold corresponds to a severe OSA criteria in children of 18 apneas/hour having a mean duration of 10 seconds. In case of control group, that threshold is fixed to be 5 apneas/hour. As a result, the following data set of labeled fragments per considered class is assessed: control (70), doubt (24), and pathologic (11), when just considering 1-hour PSG fragments, whereas the set of control (326), doubt (47) and pathologic (47) is achieved for 15-minute PSG fragments; each one also labeled according to (9).

(2) Segment Labeling. Since each taken into account is fragment of either length (one hour or 15 minutes) turns to be very long to provide computational stability when implementing discussed time-adapted PCA approach, then, PPG signals should be partitioned into processing time windows of shorter duration (termed segments). Seeing that each signal partition should comprise enough heart beats (see Figure 2), and taking into account that artifacts rarely last more than 60 seconds, then the segment length is fixed empirically to be 90 seconds. Further, every 90 -second segment is given the same label as the respective PPG fragment, wherein the partition is included. Nonetheless, there is a need for further clustering procedure to ensure that the assessed set of PPG segments are properly labeled. After carried on biclass clustering (one cluster per class, control or apneic), by using algorithm discussed in [12], distanced far enough from both cluster centroids are removed from present analysis.
TABLE 1: Amount of 90-second partitions accomplished for both cases of labeled ppg signal length.

\begin{tabular}{lcc}
\hline Clinical OSA diagnosis & \# Segments $\left(^{*}\right)$ & \# Segments $\left({ }^{* *}\right)$ \\
\hline \multicolumn{3}{c}{ Labeled PPG signal of 60-minute length } \\
\hline Normal & 2618 & 1908 \\
Pathologic & 416 & 293 \\
Assembled set & 3034 & 2201 \\
\hline \multicolumn{3}{c}{ Labeled PPG signal of 15-minute length } \\
\hline Normal & 2046 & 672 \\
Pathologic & 409 & 332 \\
Assembled set & 2455 & 1005 \\
\hline
\end{tabular}

So, the remaining group of segments adequately labeled becomes herein the training set.

Table 1 summarizes the amount of 90 -second segments accomplished for both cases of considered PPG signal length: firstly, after artifact removal $(*)$, then after clustering $(* *)$, which becomes the considered training set.

\section{Results}

4.1. TFR Enhancement and Feature Generation. Figure 3 illustrates examples of estimated enhanced TFRs that are performed for cases of normal and pathological partitions, respectively. Assessed TFRs are the matrices of dimension $T \times$ $F$, where $F$ is the number of spectral components of the PPG signal, $f=[0,1] \mathrm{Hz}$, and $T$ is the number of discrete-time samples of each recording. This arrangement is intended to cover the full-time range as well as a broad range of frequencies. As seen, the normal case holds the low frequency $(0.04-0.15 \mathrm{~Hz})$ and high frequency $(0.15-0.5 \mathrm{~Hz})$ bands of the signal. Conversely, the pathological representation does not have this high frequency component, but its energy is concentrated around the lower frequencies. Nevertheless, to illustrate the difficultness of addressed problem, Figure 3 shows several PPG segments belonging to normal (see Figures 3(a) and 3(c)), and pathological classes (see Figures 3(b) and 3(d)) along with their respective estimated TFR, and it can be seen that there are some normal segments whose waveform resembles pathological ones, and vice versa. A quantitative measure of the information contained in the TFR maps is the entropy of each band [13], with frequencies between 0.04 and $0.15 \mathrm{~Hz}$ in the low band, and frequencies between 0.15 and $0.5 \mathrm{~Hz}$ in the high band. Table 2 shows the results of the average entropy for each class as well as the average entropy for all the TFR maps, no matter what its class is.

Since the selection of the appropriate $t-f$ representation is required, tuning of the respective parameters is achieved by a procedure developed for biosignals that is discussed in [14]. Based on above explained spectral PPG envelope properties, the STFT-based quadratic spectrogram is computed by sliding Hamming windows for the following set of estimation TFR parameters: $37.5 \mathrm{~ms}$ processing window length, $50 \%$ of overlapping, and 512 frequency bins. 


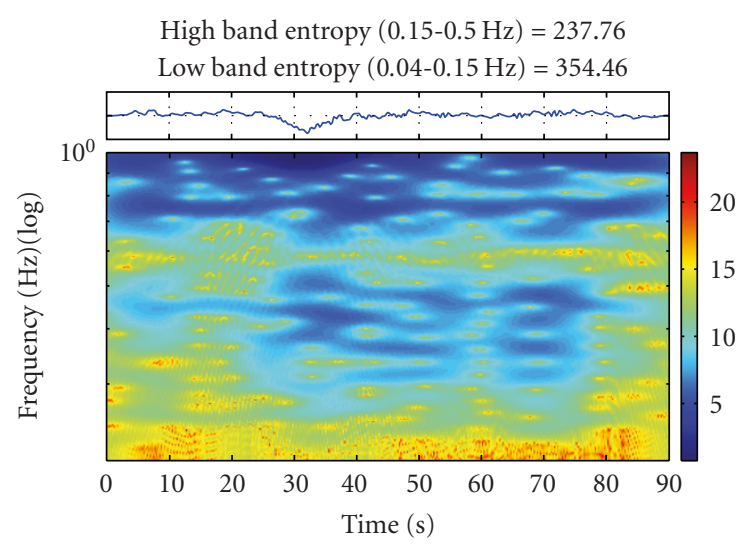

(a) Normal

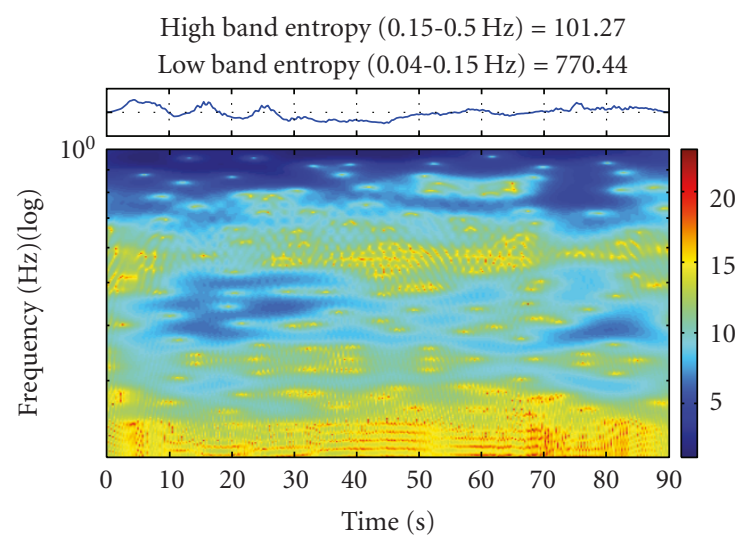

(c) Normal

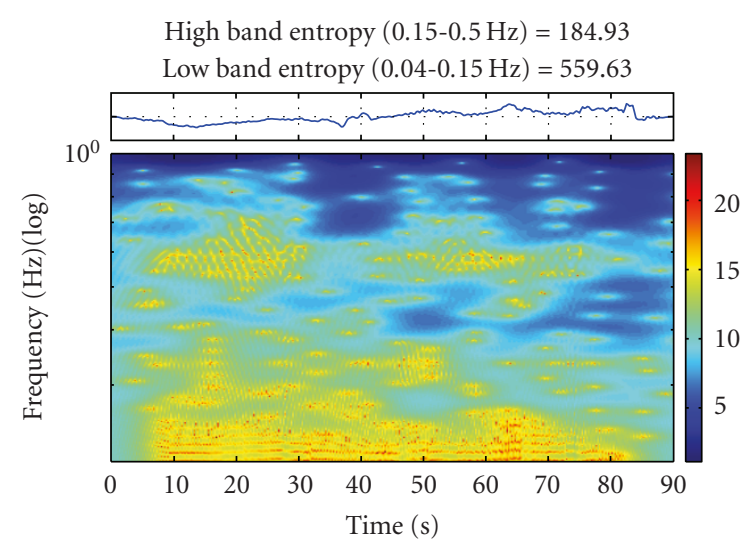

(b) Apnoea

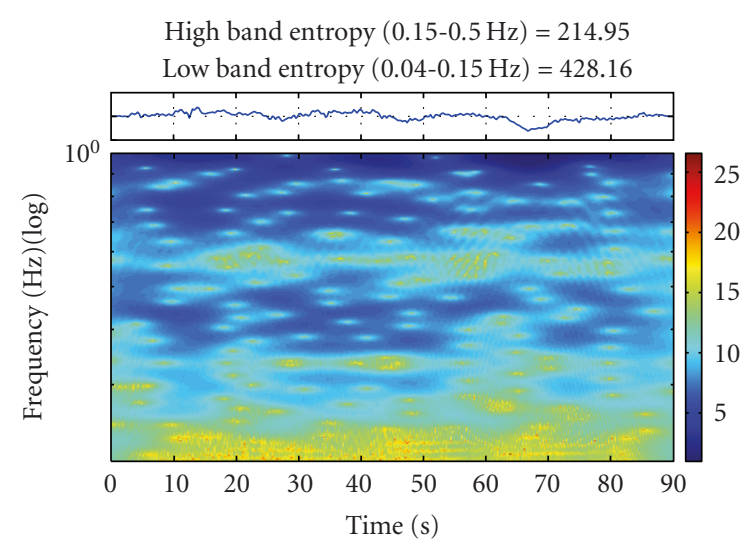

(d) Apnoea

FIgURE 3: Estimated TFR for examples of segments of 90-second length of the PPG envelope signals having labels: normal or apnoea, respectively.

4.2. Estimation of Relevance Weights of Dynamic Features. Another aspect worthy of explicit attention is the generation of TFR-based dynamic features to be under study. Specifically for the present work, procedures for computation of cepstral coefficients and centroids are similar, where in both cases each TFR is split into a fixed number of bands [14]. So, in respect to calculation of coefficients, given in (3) and (4), the following working parameters are to be determined, namely, the initial number of time-variant features, the number of bank filters, the impulse response, and its overlap over frequency domain. Nonetheless, it should be remarked that the initial number of dynamic features to be fixed is not a critical issue for the proposed training methodology since this amount is to be refined next by the relevance analysis.

Therefore, in accordance to the accuracy reached for a basic $k$-nn classifier, as shown in Figure 4, the input data space includes the following 39 TFR-based dynamic features to be further studied: the first 22 spectral centroids and their respective energy (estimated by using Hamming filters with $30 \%$ overlap, linear response distribution, and fixing $\gamma=1$ ), and the first 17 time series of vector cepstral coefficients that are computed by 48 triangular response filters with $50 \%$ overlap.
TABLE 2: Average entropy.

\begin{tabular}{lcc}
\hline Class & Frequency band & Entropy average \\
\hline Normal & High band & 316.22 \\
Normal & Low band & 651.06 \\
Pathological & High band & 291.70 \\
Pathological & Low band & 672.88 \\
Normal and Pathological & High band & 312.86 \\
Normal and Pathological & Low band & 654.05 \\
\hline
\end{tabular}

As stated above, each time-dependent feature is assumed to have a relative associated weight of relevance; the largest the estimated weight in (8) the most relevant the respective dynamic feature. However, any estimate of relevance weight is conditioned by the given dynamic feature set taken into account during calculation. Furthermore, for the concrete case of OSA diagnosing, selection of the best set of features can be achieved using, at least, two different combining approaches of comparison. Firstly, when taking a partially divided set that comprises just a single type of performed dynamic features, that is, having the same principle of generation (see (3), (4) and (5)). Secondly, when the best 


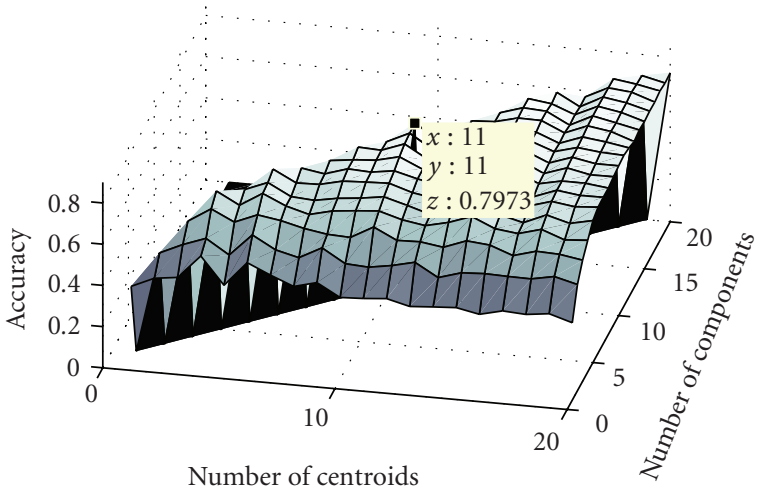

(a) Spectral centroids

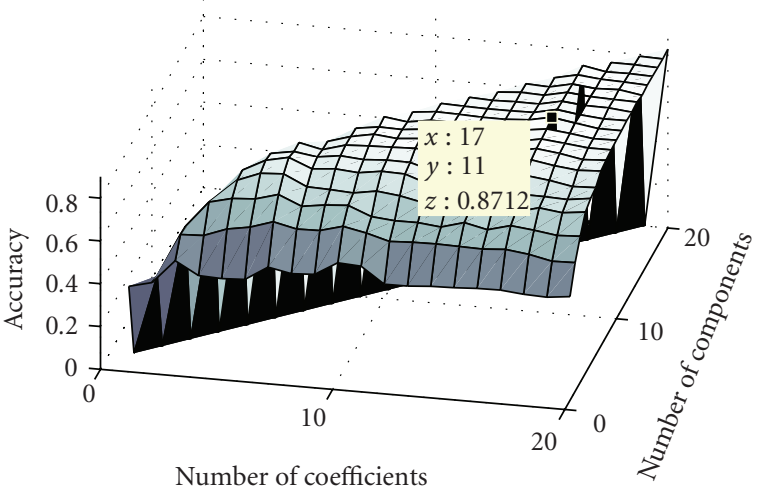

(b) Cepstral coefficients

Figure 4: On adjusting the number of TFR-based dynamic features.

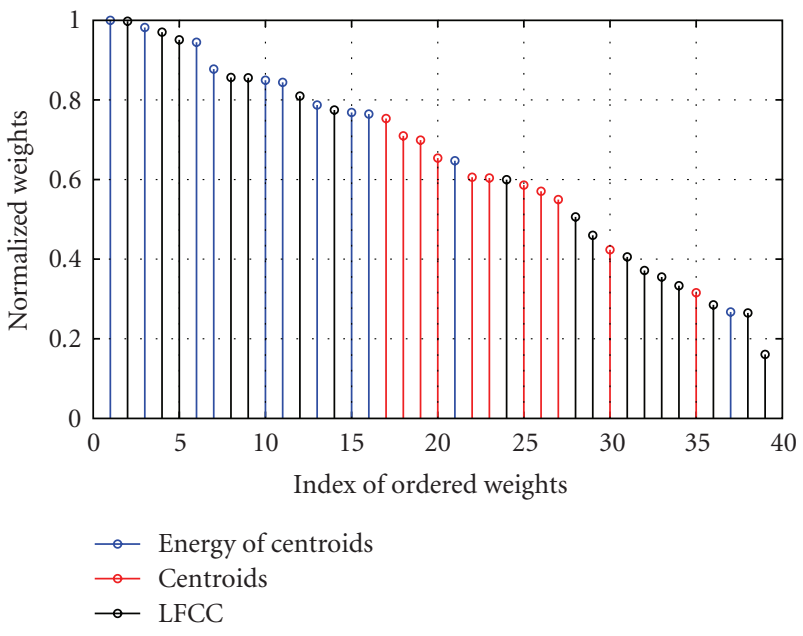

(a) Full set-based estimation

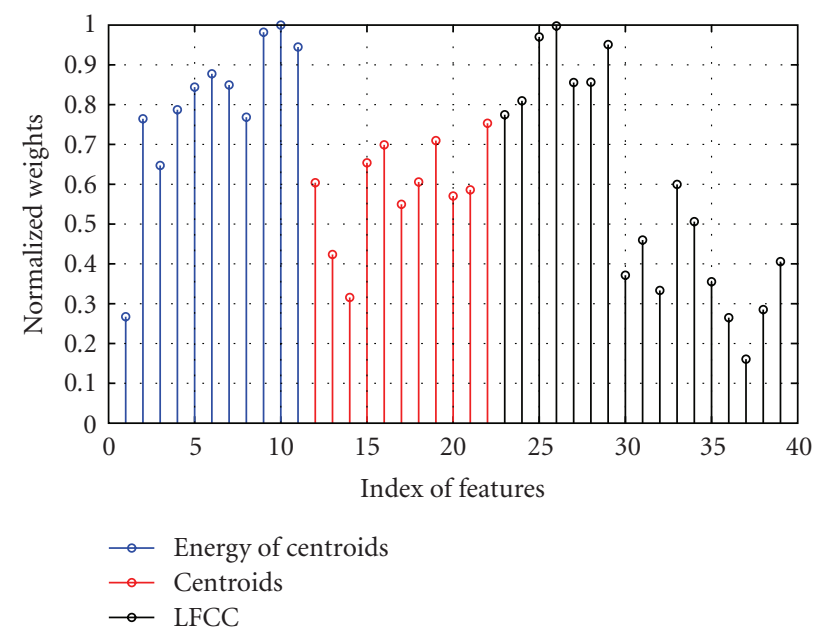

(b) Estimates for partially divided set

FIGURE 5: On computing relevance weights for considered combining approaches of comparison among dynamic features.

contours are chosen among the whole set of features no matter on their physical meaning. In this work, both combining approaches of dynamic features are studied in terms of dimension reduction, but also of accuracy performance. It must be quoted that the former approach of selection is more commonly used because of the convenient physical interpretation of selected set of features.

Nonetheless, and just for the sake of illustration, this work carries out tuning of proposed training approach based on the latter combining way since the amount of considered dynamic features is significantly the higher. Specifically, the normalized relevance weights, which are estimated according to discussed methodology of relevance analysis for stochastic processes, are depicted in Figure 5, being ordered by ordinal feature numbers, which are calculated when taking the whole set of dynamic features (see Figure 5(a)), and partially divided set (see Figure 5(b)), respectively.

4.3. Performed Classification Accuracy. Throughout the following training procedures, the metric to adjust the different schemes of considered parameterizations is the classification accuracy for the automatic OSA detection, which is estimated using a simple $k$-nearest neighbor classifier, or $k$-nn classifier. Several reasons account for the widespread use of this classifier: it is straightforward to implement, it generally leads to a good recognition performance thanks to the nonlinearity of its decision boundaries, and its complexity is assumed to be independent of the number of classes. In this concrete case, discussed methodology of training assesses the tuning of the used $k$-nn classifier by calculating its optimal number of neighbors in terms of accuracy performance, as shown in Figure 6.

With the aim of validating the discussed training methodology for OSA detection, it is desired to obtain a diagnostic over the full set of fragments to either considered length. In turn, each fragment is diagnosed to be related of either class grounded on decisions that are attained for the set of segments comprising the fragment in hand. Namely, at the beginning, there is a need to fix a minimum number of segments classified as pathologic for giving the same label to each fragment. That pathologic segment number, 
TABLE 3: Classification of PPG fragments for partially divided set.

\begin{tabular}{|c|c|c|c|c|c|c|}
\hline \multirow{2}{*}{ Dynamic feature set } & \multicolumn{3}{|c|}{ Classification for 60 -m-length } & \multicolumn{3}{|c|}{ Classification for 15-m-length } \\
\hline & $\mathrm{Se}(\%)$ & $S_{p}(\%)$ & $\operatorname{Acc}(\%)$ & $\mathrm{Se}(\%)$ & $S_{p}(\%)$ & $\operatorname{Acc}(\%)$ \\
\hline Energy of Centroids & 81.82 & 94.29 & 92.59 & 95.74 & 54.60 & 59.79 \\
\hline Centroids & 90.91 & 100 & 98.77 & 91.49 & 95.40 & 94.91 \\
\hline LFCC & 100 & 85.71 & 87.65 & 93.62 & 95.40 & 95.40 \\
\hline Full set & 100 & 100 & 100 & 97.98 & 93.56 & 93.35 \\
\hline
\end{tabular}

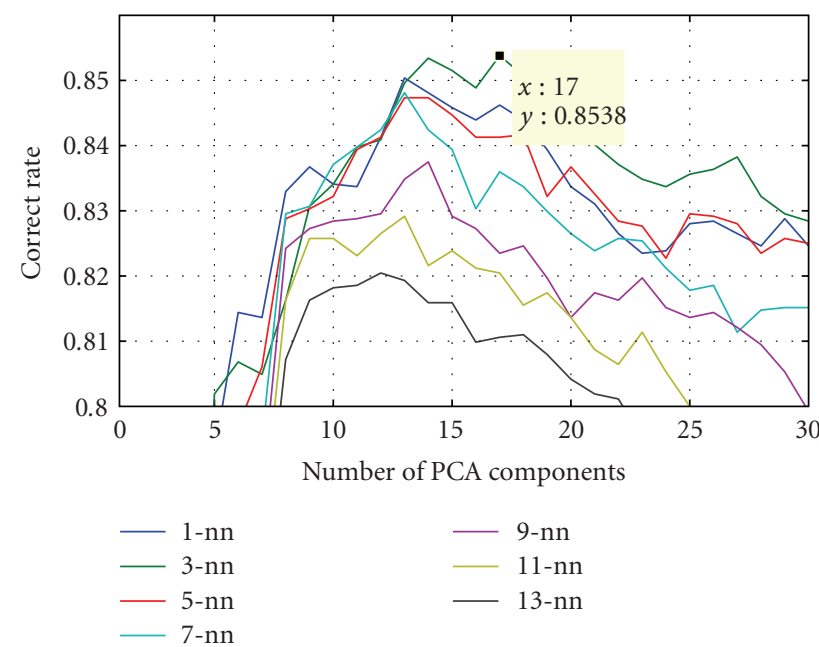

Figure 6: Tuning of $k$-nn classifier by calculating the optimal number of neighbors in terms of accuracy performance.

termed decision threshold, is fixed on dependence on both considered fragment lengths.

It should be remarked that in this work, and because of reduced input data assemble, some recordings are used for both training and validation, as well. Therefore, for testing the classifier the apparent accuracy is assessed that is performed by using $k$-nn classifier $(k=3)$, as shown in Table 3.

The decision threshold is proposed to be adjusted based on performed ROC curve for patient classification, as shown in Figure 7. So, the location where the ROC curve gets the better classification accuracy points out to the decision threshold.

Lastly, each patient is diagnosed based on those decisions made from the set of fragments measured for him. So a rule to determine when a patient with a given number of pathological fragments is considered as a pathologic subject is needed. To do this, the percentage of time under pathologic fragments was considered and this threshold was selected for maximizing Se and $\mathrm{Sp}$, ratio at the ROC curve.

Table 4 summarizes the performed patient classification accuracy for both considered combining approaches of dynamic features (partial and full set). In accordance with the discussed approach of relevance analysis, the LFCC and Centroids subsets of dynamic features reach the better accuracy that is similar to the one achieved for the whole training set. As a result, both sets should be strongly

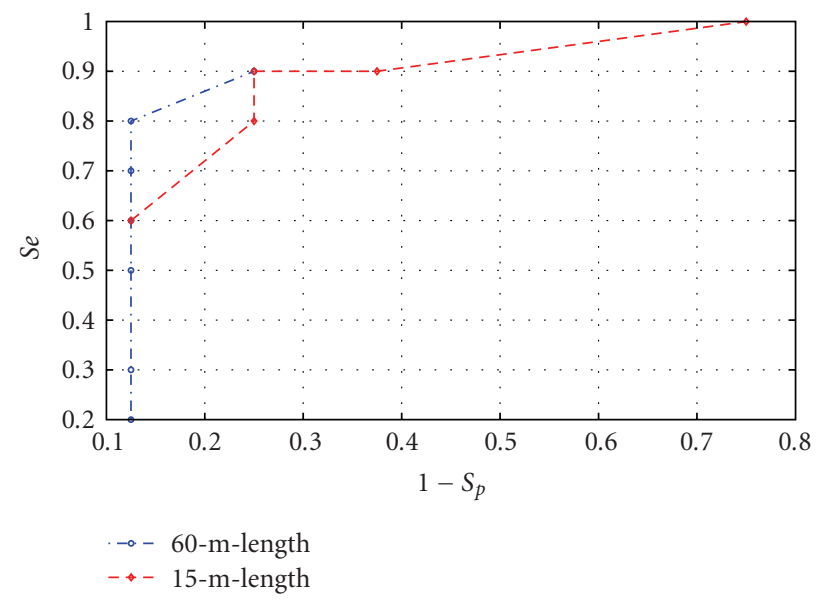

Figure 7: Performed ROC curves on dependence on both considered fragment lengths.

TABLE 4: Classification of patient for training based on partially divided set of dynamic features.

\begin{tabular}{lccc}
\hline Dynamic feature set & $S e(\%)$ & $S_{p}(\%)$ & $A c c(\%)$ \\
\hline Energy of Centroids & 70.00 & 87.50 & 73.68 \\
Centroids & 80.00 & 87.50 & 83.33 \\
LFCC & 90.00 & 75.00 & 83.33 \\
Full set & 80.00 & 87.50 & 83.33 \\
\hline
\end{tabular}

considered for OSA diagnosing with the advantage that the each performed time-evolving parameter is related to a fixed spectral subband, and thus, leading to easer clinical interpretation. It must be quoted that displayed outcomes of accuracy in Table 4 are performed just when considering training over 60-m-length fragments. In case of 15 -m-length, and if taking into consideration the full set of dynamic features, the overall performance is the following: $S e=$ $90 \%, S_{p}=62.5 \%$, and $A c c=77.78 \%$, which is significatively lower that those assessed outcomes for training over $60-\mathrm{m}$ length fragments.

Next, the energy subset shows high relevance, but a low performance; this may be explained because of notable redundance among the features. Therefore, the set of energies that is described by (5) should be rejected as perspective dynamic features for OSA diagnosing. 


\section{Discussion}

It should be remarked that the main goal of present paper is to use a complex of signal processing algorithms for the improvement in OSA diagnosis from PPG recordings, as an alternative for sleep apnea screening with the added benefit of low cost and simplicity. The methodology lies on the hypothesis that each time-dependent characteristic holds a relative associated weight of relevance, and in this connection, the results also evidence the following aspects to take into consideration.

(i) The enhanced parameter estimation carried out by introducing $t-f$ representations should be regarded as a remarkable factor for an adequate generation of any set of dynamic features. Here, feature enhancement is performed by means of nonparametric spectrogram-based TFR that had been reported to be appropriate for the analysis of nonstationary biological signals consisting of different frequency components. Nonetheless, for the discussed methodology for OSA detection, needed TFR enhancement for dynamic feature extraction can be performed by using more elaborated approaches: wavelet-based scalograms, projection pursuit, by using time frequency distributions, and so forth, as discussed in [14]. Yet, no matter which particular TFR estimation method is used, the final result is a large data matrix containing the time-frequency pattern, which has to be transformed into a feature vector for classification purposes holding the most relevant information in a compact fashion.

(ii) With regard to feature extraction and selection, proposed methodology for relevance analysis of dynamic relevance is based on time-adapted linear component approach. At this point, two main issues are to be considered: the measure associated to a given relevance function, and the multivariate transformation through the time axis, which is assumed to maximize the measure of relevance present in the contours by their projection onto a new space. As a measure of relevance, the maximum variance is assumed. Specifically, time-adapted PCA version is discussed throughout this paper as unsupervised method to perform relevance analysis of considered set of stochastic features. Though proposed methodology of relevance analysis can extended to other techniques linear component decomposition, as shown in [15].

(iii) Two different combining approaches for selecting the best set of contours are studied. Firstly, when taking a partially divided set that relates dynamic features having the same principle of generation. Secondly, when the best features are chosen despite of their physical meaning. From performed accuracy showed in Table 3 one can conclude that even that the former case reaches comparable figures of accuracy, the latter approach of selection is more commonly used because of the convenient physical interpretation of selected set of features. Furthermore, it has been established that the set of LFCC dynamic features should be strongly considered for OSA diagnosing. Performed outcomes bring enough evidence that if using a subset of LFCC features a fragment classification accuracy can reach as much as $93 \%$ value, which provides an adequate scheme for ambulatory OSA diagnosis. Therefore, to take into account evolution of random biological variables along time, definitively, leads to an accuracy improvement of OSA detection. Nonetheless, more efforts might be done to define feature set carrying fundamental information for the OSA classification, as quoted in [16]. Though, performed outcomes look very promising in terms of accuracy of features extraction, testing of the discussed methodology should be provided using larger data sets.

(iv) The set of considered pathological subjects shows a larger low frequency entropy than the set of normals as expected from the bigger envelope oscillations driven by apnea. The reverse happens when analyzing entropy in the high frequency band where pathologic subjects reduce the entropy as compared to normals.

(v) The discussed automated system for OSA diagnosing is based on analysis of set of fragments that are partitioned from the PPG envelope recordings. In this regard, labeling of partitioned PPG envelope recordings is provided so to have time epochs identified as apneic or not apneic. However, in clinical practice usually the interest lies in having a subject diagnosis related to apnea, both in adults [17] and children [4], and not just a time screening of the apnea events. With this aim, a rule has been applied to the fragment labeling, providing subject specific diagnosis. Comparison with PSG clinical decision is provided, showing the potential of the methods here presented. As a result, PPG can be considered as a promising alternative to reduce the number of the PSG sleep recordings.

\section{Conclusions}

A new methodology for OSA detection is explored, which is based on relevance analysis of dynamic features extracted from nonparametric $t-f$ representation of the recordings of PPG envelope. Particularly, a time-evolving version of the standard PCA is discussed that performs stochastic dimensionality reduction of the dynamic features in hand. Discussed methodology of relevance analysis benefits of the dynamic properties of the time-evolving spectral parameters, during either transient physiological or pathological episodes. As a result, PPG can be considered as a promising alternative to reduce the number of the PSG sleep recordings.

In addition, two different combining approaches for selecting the best set of contours are studied: firstly, when taking dynamic features having the same principle of generation. Secondly, when the best features are chosen despite of their physical meaning. In this case, the latter 
approach turns to be more suitable because of the convenient physical interpretation of selected set of features and provided accuracy of selection is more commonly used because of the convenient physical interpretation of selected set of features. Furthermore, it has been established that the LFCC and Centroids subsets of dynamic features should be strongly considered for OSA diagnosing since it increases the specificity in the apnoea detector. Both subsets display a patient classification accuracy of $83.33 \%$, while in [4] an accuracy of $80 \%$ is reported; consequently, the advantage of the method proposed in this paper to increase the specificity of the obstructive sleep apnea detector is evident.

The TFR-based parameter estimation is a remarkable factor for an adequate dynamic feature generation. Therefore, for OSA detection, it would be of benefit to explore needed enhancement by using more elaborated approaches (waveletbased scalograms, matching pursuit, etc.). Besides, as feature work, further efforts on finding an alternative for OSA diagnosing, having the added benefit of low cost and simplicity, should be focused on extended studies to corroborate the potential of another approaches in conjunction with heart rate variation analysis $[18,19]$.

\section{Acknowledgments}

This work is supported by the Ministerio de Ciencia y Tecnología, FEDER, under project TEC2010-21703-C03-02, by CIBER de Bioingeniería, Biomateriales y Nanomedicina through Instituto de Salud Carlos III, by ARAID and Ibercaja under project "Programa de APOYO A LA I+D+i" by Grupo Consolidado GTC from DGA (Spain), and by "Centro de Investigación e Innovación de Excelencia-ARTICA", financed by COLCIENCIAS (Colombia) y Becas para Estudiantes Sobresalientes de Posgrado de la Universidad Nacional de Colombia.

\section{References}

[1] W. W. Flemons, D. Buysse, S. Redline et al., "Sleep-related breathing disorders in adults: recommendations for syndrome definition and measurement techniques in clinical research," Sleep, vol. 22, no. 5, pp. 667-689, 1999.

[2] E. Gil, J. María Vergara, and P. Laguna, "Detection of decreases in the amplitude fluctuation of pulse photoplethysmography signal as indication of obstructive sleep apnea syndrome in children," Biomedical Signal Processing and Control, vol. 3, no. 3, pp. 267-277, 2008.

[3] E. Gil, M. Mendez, J. M. Vergara, S. Cerutti, A. M. Bianchi, and P. Laguna, "Discrimination of sleep-apnea-related decreases in the amplitude fluctuations of ppg signal in children by HRV analysis," IEEE Transactions on Biomedical Engineering, vol. 56, no. 4, pp. 1005-1014, 2009.

[4] E. Gil, R. Bailón, J. M. Vergara, and P. Laguna, "PTT variability for discrimination of sleep apnea related decreases in the amplitude fluctuations of PPG signal in children," IEEE Transactions on Biomedical Engineering, vol. 57, no. 5, pp. 1079-1088, 2010.

[5] M. Sun, M. L. Scheuer, and R. J. Sclabassi, "Decomposition of biomedical signals for enhancement of their time-frequency distributions," Journal of the Franklin Institute, vol. 337, no. 4, pp. 453-467, 2000.

[6] S. Cerutti, "In the spotlight: biomedical signal processing: a well established discipline or a paradigm to promising integrated visions?" IEEE Reviews in Biomedical Engineering, vol. 2, pp. 9-11, 2009.

[7] Y. Zhao and S. Zhang, "Generalized dimension-reduction framework for recent-biased time series analysis," IEEE Transactions on Knowledge and Data Engineering, vol. 18, no. 2, pp. 231-244, 2006.

[8] G. M. Loughlin, R. T. Brouillette, L. J. Brooke et al., "Standards and indications for cardiopulmonary sleep studies in children," American Journal of Respiratory and Critical Care Medicine, vol. 153, no. 2, pp. 866-878, 1996.

[9] C. L. Marcus, R. D. Annett, L. J. Brooks et al., "Cardiorespiratory sleep studies in children: establishment of normative data and polysomnographic predictors of morbidity," American Journal of Respiratory and Critical Care Medicine, vol. 160, no. 4, pp. 1381-1387, 1999.

[10] C. L. Marcus, "Sleep-disordered breathing in children," American Journal of Respiratory and Critical Care Medicine, vol. 164, no. 1, pp. 16-30, 2001.

[11] J. Allen, "Photoplethysmography and its application in clinical physiological measurement," Physiological Measurement, vol. 28, no. 3, pp. R1-R39, 2007.

[12] T. Kanungo, D. M. Mount, N. S. Netanyahu, C. D. Piatko, R. Silverman, and A. Y. Wu, "An efficient k-means clustering algorithms: analysis and implementation," IEEE Transactions on Pattern Analysis and Machine Intelligence, vol. 24, no. 7, pp. 881-892, 2002.

[13] R. G. Baraniuk, P. Flandrin, A. J. E. M. Janssen, and O. J. J. Michel, "Measuring time-frequency information content using the Rényi entropies," IEEE Transactions on Information Theory, vol. 47, no. 4, pp. 1391-1409, 2001.

[14] A. F. Quiceno-Manrique, J. I. Godino-Llorente, M. BlancoVelasco, and G. Castellanos-Dominguez, "Selection of dynamic features based on time-frequency representations for heart murmur detection from phonocardiographic signals," Annals of Biomedical Engineering, vol. 38, no. 1, pp. 118-137, 2010.

[15] L. D. Avendaño-Valencia, J. I. Godino-Llorente, M. BlancoVelasco, and G. Castellanos-Dominguez, "Feature extraction from parametric time-frequency representations for heart murmur detection," Annals of Biomedical Engineering, vol. 38, no. 8, pp. 2716-2732, 2010.

[16] M. O. Mendez, J. Corthout, S. van Huffel et al., "Automatic screening of obstructive sleep apnea from the ECG based on empirical mode decomposition and wavelet analysis," Physiological Measurement, vol. 31, no. 3, pp. 273-289, 2010.

[17] A. H. Khandoker, M. Palaniswami, and C. K. Karmakar, "Support vector machines for automated recognition of obstructive sleep apnea syndrome from ECG recordings," IEEE Transactions on Information Technology in Biomedicine, vol. 13, no. 1, pp. 37-48, 2009.

[18] M. J. Lado, X. A. Vila, L. Rodríguez-Liñares, A. J. Méndez, D. N. Olivieri, and P. Félix, "Detecting sleep apnea by heart rate variability analysis: assessing the validity of databases and algorithms," Journal of Medical Systems. In press.

[19] L. M. Sepúlveda-Cano, C. M. Travieso-González, J. I. GodinoLlorente, and G. Castellanos-Domínguez, "On improvement of detection of Obstructive Sleep Apnea by partial least squarebased extraction of dynamic features," in Proceedings of the 32nd Annual International Conference of the IEEE Engineering in Medicine and Biology Society (EMBC '10), pp. 6321-6324, Buenos Aires, Argentina, August-September 2010. 